\title{
Prediction of Maintenance of Sinus Rhythm Using Heart Rate Variability Characteristics after Conversion in Patients with Atrial Fibrillation
}

\author{
G Varoneckas, A Martinkenas, R Paskeviciute, A Podlipskyte, D Zemaityte \\ Institute of Psychophysiology and Rehabilitation, Palanga, Lithuania
}

\begin{abstract}
The goal was to find out the informative characteristics of heart rate variability (HRV) after the conversion of atrial fibrillation (AF) for prediction of maintenance of sinus rhythm (SR). HRV parameters were obtained in two ways: from Poincare plots and from spectral analysis. Sustained sinus rhythm patient group displayed significantly higher $H R V$ with no difference in baseline HR frequency calculated from Poincare plot, but not from power spectrum. A model based on binary logistic regression of HRV parameters calculated from both models for prediction of patients groups with sustained sinus rhythm or broken one was created. An increased HRV (SDRR) and a decreased minimal and an increased maximal RR intervals on Poincare plot as well as increased HFC, decreased VLFC and relative LFC from power spectrum, might be used for prediction of maintenance of $S R$.
\end{abstract}

\section{Introduction}

Atrial fibrillation (AF), a commonly encountered arrhythmia, has been the subject of increased interest and intensive clinical research in recent years [1]. AF is poorly tolerated disorders due to unpleasant complaints, like feelings of palpitation or irregularity. Irregular ventricular response and inappropriately rapid heart rate (HR) disturbing hemodynamics can lead progressively to the development of cardiomyopathy with symptoms and signs of heart failure as well as to the most usual causes of thrombi-embolic events [2]. Because of that AF is associated with more hospital admissions than any other cardiac disease. So it is very important to diagnose and to treat these arrhythmias.

The autonomic control in the pathogenesis of AF plays an important role which has not yet been clearly investigated. Analysis of heart rate variability (HRV) has emerged as a valuable noninvasive tool for assessment of autonomic HR control having predictable value in followup [3]. It has become clear that cardiovascular diseases are associated with typical changes in HRV [3]. Recent reports have indicated that a transient augmentation of sympathetic tone or alterations in parasympathetic activity may be important in the onset of AF [2]. We hypothesized that the presence of autonomic dysfunction and reduced HRV after sinus rhythm restoration could be associated with recurrent AF. It seems that HRV pattern recorded after the conversion can be useful tool for prediction of sinus rhythm maintenance.

The goal was to find out the informative characteristics of heart rate variability (HRV) after the conversion of AF for prediction of maintenance of sinus rhythm.

\section{Methods}

The contingent was 46 pts: 24 (52\%) females and 22 (48\%) males (Table 1). Most frequent causes of AF were hypertension, coronary artery disease, mitral valve disease, heart failure, thyrotoxicosis, and diabetes did not differ significantly in groups of patients with sustained sinus rhythm and broken one (Table 1). All patients with $\mathrm{AF}$ underwent convenient clinical examination, echocardiography and ECG monitoring with computerized HRV analysis in the Intensive Care Unit.

The AF was converted into the sinus rhythm by antiarrhythmic drugs (Quinidine, Amiodarone, Propafenone) in 24 patients (52.2\% of totals) and by defibrillation anywhere in 22 patients $(47.8 \%)$. During 1month follow-up the sinus rhythm was broken in 20 patients: 11 patients (45.8\%) from drug conversion group and in 9 patients $(40.9 \%)$ from defibrillation one.

HRV parameters were obtained in two ways: by construction of Poincare plots and by spectral analysis of RR intervals recorded during 2 hours after the conversion.

Poincare plots were constructed from RR intervals $\left(R_{n+1}\right)$ versus the previous intervals $\left(R_{n}\right)$, plotted as a function. The scatterplot length $(\mathrm{L})$, the minimal $\left(\mathrm{RR}_{\min }\right)$ and the maximal $R R$ interval $\left(R_{\text {max }}\right)$ representing $H R$ responses, the width (W) reflecting maximal HR variability, and the square (S), representing overall HR variability associated with total autonomic control as well as mean RR interval and its standard deviation (SDRR) were calculated (Figure 1). The scatterplot length (L) was calculated as a difference on plot diagonal between 
minimal $\left(\mathrm{RR}_{\min }\right)$ and maximal $\left(\mathrm{RR}_{\max }\right) \mathrm{RR}$ interval values. The scatterplot width $(\mathrm{W})$ was calculated as a maximal width-difference between of two points at parallel tangential lines determining the plot (see $\Delta R_{t}$ in Figure 1). The scatterplot length reflects the influence of reflex autonomic control, while scatterplot width reflects the tonic control. The ratio between reflex and tonic autonomic control $(\mathrm{D}=\mathrm{L} / \mathrm{W})$ and the scatterplot square, representing overall HR variability, were calculated too.

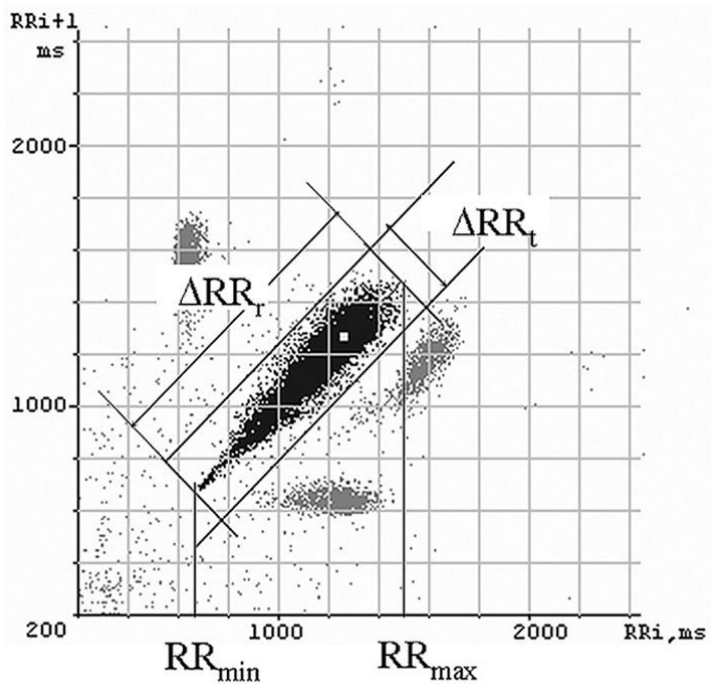

Figure 1. Quantitative analysis of HR variability by means of Poincare plots of $R R$ intervals: $R^{\min }$, minimal value of $R R$ interval; $R R_{\max }$, maximal value of $R R$ interval; $\Delta \mathrm{RR}_{\mathrm{r}}$, maximal $\mathrm{HR}$ response $(\mathrm{L}) ; \Delta \mathrm{RR}_{\mathrm{t}}$, maximal HR variability $(W)$.

Table 1. Demographics and Clinical Characteristics.

\begin{tabular}{lll}
\hline Variable & $\begin{array}{l}\text { Patients } \\
\text { with sustained } \\
\text { sinus rhythm } \\
\mathrm{n}=26\end{array}$ & $\begin{array}{l}\text { Patients } \\
\text { with broken } \\
\text { sinus rhythm } \\
\mathrm{n}=20\end{array}$ \\
\hline $\begin{array}{l}\text { Mean age, yr } \pm \text { SD } \\
\text { Gender, } \mathrm{n}(\%)\end{array}$ & $62.2 \pm 8.5$ & $66.4 \pm 9.8$ \\
Male & $8(30.8)$ & $14(70.0)$ \\
Female & $18(69.2)$ & $6(30.0)$ \\
Underlying disease & & \\
Arterial hypertension & $22(84.6)$ & $16(80.0)$ \\
Diabetes mellitus & $4(15.4)$ & $3(15.0)$ \\
Coronary artery disease & $25(96.2)$ & $19(95.0)$ \\
Mitral valve disease & $3(11.5)$ & $1(5.0)$ \\
Tyreotoxicosis & $2(7.7)$ & $2(10.0)$ \\
CHF & $25(96.2)$ & $20(100.0)$ \\
NYHA Functional Class & & \\
I & $2(7.7)$ & $14(70.0)$ \\
II & $24(92.3)$ & $5(25.0)$ \\
III & 0 & $1(5.0)$ \\
\hline
\end{tabular}

HR power spectrum analysis was performed by means of quick Furje transform, using Tucki window for interpolation of the sequence of RR intervals. HR power spectrum was broken into the four main oscillatory components: (1) ultra-low frequency component (ULFC), reflecting low frequency trends, including body movements; (2) very-low frequency component (VLFC), associated with thermal-metabolic regulation; (3) low frequency component (LFC), reflecting predominantly sympathetic control; and (4) high frequency component (HFC), reflecting parasympathetic one. The relative values of each component after the normalization with respect to the total spectrum power (SDRR) were obtained for ULFC (NULFC), VLFC (NVLFC), LFC (NLFC) and HFC (NHFC). Total power of HR spectrum was used as a marker of overall autonomic HR control.

\subsection{Statistical analysis}

Prior to statistical testing, each of the above mentioned descriptors was tested for normality. Statistical comparison of the results was performed between patient's groups with sustained sinus rhythm and broken one. To determine the significance of the differences between the means (continuous measures) Student's t-test was used. The nonparametric Mann-Whitney rank sum test was used in cases where the studied items had a nonnormal distribution. Results were presented as means (standard deviation) or medians (25-75 percentiles). The $\chi 2$ test with a Yates correction or Fisher exact test was used for the comparison of proportions between groups. A value of $p<0.05$ was considered statistically significant.

A model based on binary logistic regression of HRV parameters calculated separately from Poincare plot and from power spectrum parameters for prediction of patient's groups with sustained sinus rhythm or broken one was created. Backward Stepwise logistic regression analysis, using Wald test statistics was employed with $\mathrm{p}$ to removal 0.1 . Statistically significant predictors were obtain of odds ratio (OR) with $95 \%$ CI. P-value below 0.05 was assumed to indicate significance.

Computation was done using SPSS 11.5 statistical software.

\section{Results}

\subsection{HRV parameters in patient groups with sustained and broken sinus rhythm during one-month follow-up}

HR frequency during two-hour period after the conversion of $\mathrm{AF}$ into the sinus rhythm did not differ significantly in both groups of patients, with sustained sinus rhythm and with broken one during one-month follow-up (Table 2). Although patients with sustained 
sinus rhythm demonstrated higher HRV calculated from Poincare plots, but not from spectral analysis. Analysis of Poincare plots of RR intervals have shown that HRV (SDRR), maximal value of $R R$ interval $\left(R_{\max }\right)$, scatterplot width (W) and square (S) were significantly higher in group with sustained sinus rhythm, except the ratio between reflex and tonic autonomic control (D), which was higher in group with broken sinus rhythm. Power spectral analysis demonstrated that total power and all of oscillatory components were decreased, but not statistically significant, in patient group with broken sinus rhythm. The higher HRV in patient with sustained sinus rhythm is clearly demonstrated in Poincare plot presented in Figure 2.
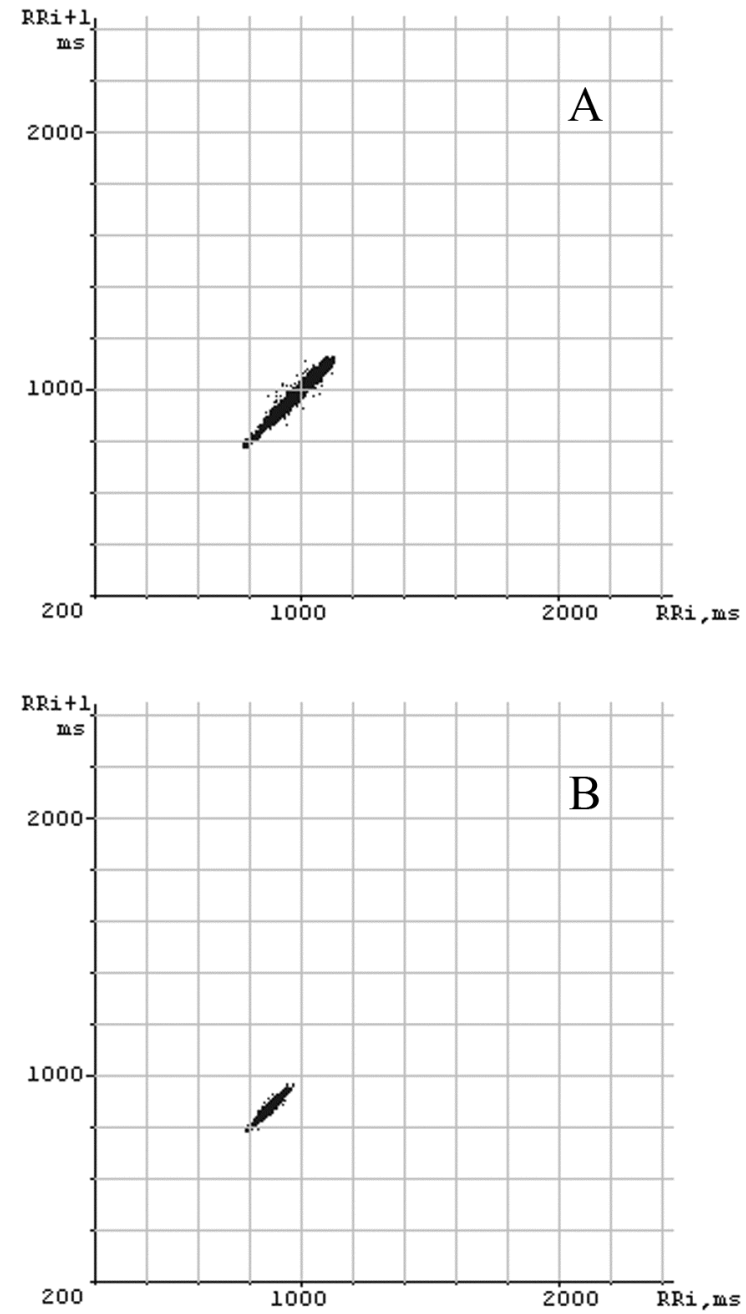

Figure 2. Poincare plots of RR intervals after the conversion in sinus rhythm in patient with sustained sinus rhythm (A) and in patient with broken sinus rhythm (B) during 2-month follow-up.

\subsection{Models based on binary logistic regression of HRV parameters for prediction of sinus rhythm maintenance}

HRV parameters obtained from Poincare plot versus HRV parameters obtained from spectral analysis were used for elaboration of the binary logistic regression models for prediction of maintenance of sinus rhythm (see Table 2).

Modelling for prediction of patient groups with sustained sinus rhythm and broken one based on HRV parameters from Poincare plots demonstrated statistically significant full model versus a model with intercept only (omnibus effect of scenario), $\chi^{2}(4, \quad \mathrm{~N}=46)=16.62$, $\mathrm{p}=0.002$. $-2 \log$ Likelihood statistics was 46.31. The Nagelkerke $R^{2}=0.41$. The most significant independent predictors for sustained sinus rhythm were: increased SDRR an odds ratio of 1.13 for each increasing unit $(\mathrm{p}=$ $0.034,95 \%$ CI $1.01-1.27)$, decreased $R_{\text {min }}$ an OR 0.95 ( $\mathrm{p}=0.006,95 \%$ CI $0.92-0.99)$, and increased RRmax an OR 1.05 ( $\mathrm{p}=0.005,95 \%$ CI $1.02-1.09$ ).

The model was able correctly to classify $71.7 \%, 80.8 \%$ sensitivity for patients with sustained sinus rhythm and $60.0 \%$ specificity for patients with broken one (Table 3 ).

Table 2. HRV parameters, calculated from Poincare plot and power spectrum in patients with sustained sinus rhythm and broken one during 1-month follow-up.

\begin{tabular}{lccc}
\hline $\begin{array}{l}\text { HRV } \\
\text { parameters } \\
\text { by means of }\end{array}$ & $\begin{array}{c}\text { Patients with } \\
\text { sustained sinus } \\
\text { rhythm }(n=26)\end{array}$ & $\begin{array}{c}\text { Patients with } \\
\text { broken sinus } \\
\text { rhythm }(n=20)\end{array}$ & $\begin{array}{c}\mathrm{p} \\
\text { value }\end{array}$ \\
\hline Poincare plots & & & \\
RR; ms & $910.4 \pm 150.6$ & $824.9 \pm 179.3$ & $\mathrm{~ns}$ \\
SDRR; ms & $46.4 \pm 23.9$ & $31.6 \pm 18.8$ & 0.027 \\
RR $_{\min } ; \mathrm{ms}$ & $777.4 \pm 126.4$ & $728.8 \pm 141.5$ & $\mathrm{~ns}$ \\
RR $_{\max } ; \mathrm{ms}$ & $1032.9 \pm 188.1$ & $890.9 \pm 205.2$ & 0.019 \\
W; ms & $268.3 \pm 111.1$ & $170.9 \pm 96.7$ & 0.003 \\
L; ms & $73.8 \pm 26.0$ & $57.7 \pm 32.8$ & $\mathrm{~ns}$ \\
D; $\%$ & $29.3 \pm 8.0$ & $38.7 \pm 17.2$ & 0.018 \\
S; ms & 11040 & 7504 & 0.03 \\
Range & $(8096-17920)$ & $(2172-11278)$ & \\
\hline Spectral analysis & & & \\
RR; ms & $910.4 \pm 150.6$ & $824.9 \pm 179.3$ & $\mathrm{~ns}$ \\
SDRR; ms & $49.1 \pm 27.3$ & $34.9 \pm 19.7$ & $\mathrm{~ns}$ \\
ULFC; ms & $27.8 \pm 16.8$ & $19.3 \pm 14.4$ & $\mathrm{~ns}$ \\
VLFC; ms & $30(21-45)$ & $23(17-33)$ & $\mathrm{ns}$ \\
LFC; ms & $13.6 \pm 7.9$ & $9.7 \pm 9.0$ & $\mathrm{~ns}$ \\
HFC; ms & $8(4-13)$ & $8(6-11)$ & $\mathrm{ns}$ \\
NULFC; $\%$ & $30.6 \pm 8.3$ & $29.1 \pm 12.4$ & $\mathrm{~ns}$ \\
NVLFC; $\%$ & $41.1 \pm 9.5$ & $39.6 \pm 8.7$ & $\mathrm{~ns}$ \\
NLFC; $\%$ & $15.7 \pm 5.6$ & $13.8 \pm 6.2$ & $\mathrm{~ns}$ \\
NHFC; $\%$ & $10(8-17)$ & $13(10-23)$ & $\mathrm{ns}$ \\
\hline N & &
\end{tabular}

Means \pm SD or medians (25-75 percentile); $p$ value between groups; ns - no significant difference between groups. Used Student's t-test or the Mann-Whitney test when appropriate. 
Modelling for prediction of patient groups with sustained sinus rhythm and broken one based on HRV parameters from spectral analysis demonstrated statistically significant full model versus a model with intercept only, $\chi^{2}(3, \quad \mathrm{~N}=37)=9.67, \quad \mathrm{p}=0.022 . \quad-2 \log$ Likelihood statistics was 40.95 . The Nagelkerke $\mathrm{R}^{2}=0.31$. The most significant independent predictors for sustained sinus rhythm were: increased HFC an OR $1.14(\mathrm{p}=0.084$, $95 \%$ CI 0.98 - 1.32), decreased VLFC an OR 0.94 $(\mathrm{p}=0.038,95 \%$ CI $0.88-1.00)$, and NLFC an OR 0.81 $(\mathrm{p}=0.041,95 \%$ CI $0.66-0.99)$.

The prediction of sinus rhythm maintenance based on power spectrum parameters was correctly classified with overall accuracy of $73 \%$ (sensitivity $71.4 \%$ and specificity $75 \%$ ) (Table 3 ).

Table 3. Accuracy of two models for prediction of sinus rhythm maintenance based on HRV parameters obtained from Poincare plots or from power spectrum.

\begin{tabular}{lcc}
\hline Accuracy & \multicolumn{2}{c}{ Models based on HRV parameters } \\
\cline { 2 - 3 } & Poincare plots & Power spectrum \\
\hline Sensitivity; \% & 80.8 & 71.4 \\
Specificity; \% & 60.0 & 75.0 \\
False Positive Rate; \% & 27.5 & 21.5 \\
False Negative Rate; \% & 41.7 & 33.3 \\
Overall correct; \% & 71.7 & 73.0 \\
\hline
\end{tabular}

$\mathrm{p}>0.05$ between groups

\section{Discussion}

The many risk factors such as old age, male sex, history of hypertension, myocardial ischemia, decreased left ventricular function, diabetes and modifications of autonomic control are associated with the development of atrial fibrillation [1, 3]. Our data regarding autonomic control suggest that an increased HRV reflecting a higher vagal influence predicts sinus rhythm; while a decreased one reflecting augmented sympathetic tone is associated with repetitive AF.

The groups of patients with maintained sinus rhythm and broken one statistically differ only by HRV parameters obtained from Poincare plots displaying nonsignificant difference in baseline HR frequency. It means that HR pattern presented in Poincare plot (time domain in two-dimensional scaling) but not in power spectrum (frequency domain parameters) are more informative for prediction of sinus rhythm maintenance after the conversion of AF.
The both created models based on binary logistic regression of HRV parameters obtained by different methods, Poincare plot and power spectrum, demonstrated appropriateness for prediction of sinus rhythm maintenance with no significant difference in accuracy between them.

\section{Conclusions}

Significantly higher HRV with no difference in baseline HR frequency calculated from Poincare plot of RR intervals, but not from power spectrum, was found in sustained sinus rhythm patient group, as compared with patients with broken one.

Models based on binary logistic regression of HRV parameters for prediction of sinus rhythm maintenance demonstrated that an increased total HRV (SDRR) and a range from minimal to maximal $R R$ interval on Poincare plot characterizing the HR responses as well as increased HFC, decreased VLFC, and diminished NLFC calculated from power spectrum were the most significant independent predictors for sustained sinus rhythm.

HRV parameters might be useful for prediction of maintenance of sinus rhythm after the conversion in patients with atrial fibrillation.

\section{References}

[1] Fuster V, Rydén LE, Asinger RW, Cannom DS, Crijns HJ, Frye RL, Halperin JL, Kay GN, Klein WW, Lévy S, McNamara RL, Prystowsky EN, Wann LS, Wyse DG. Atrial Fibrillation. Report from the Joint Task Force of the ESC, the ACC and the AHA. European Heart Journal 2001, 22:1852-923.

[2] Vardas PE, Manios EG, Kanoupakis EM, Mavrakis HE. Temporary internal atrial defibrillation in patients with atrial fibrillation: conclusions from the use of the latest technology. From I.E. Ovsyshcher Cardiac Arrhythmias and Perspectives for the New Century, New York: Futura, 2000:53-62.

[3] Van den Berg MP, Haaksma J, Brouwer J, Tieleman RG, Mulder G, Crijns HJG.M. Heart rate variability in patients with atrial fibrillation is related to vagal tone. Circulation 1997, 96:1206-16.

Address for correspondence:

Giedrius Varoneckas

Institute of Psychophysiology and Rehabilitation

Vyduno Str. 4, Palanga LT-00135, Lithuania

E-mail: giedvar@ktl.mii.lt 